\title{
论 文核能领域关键力学问题专题
}

\section{芯体多孔结构对单片式燃料元件辐照热-力耦合 行为的影响研究}

严峰 $^{1}$ ，孔祥喆 ${ }^{1}$ ，丁淑蓉 ${ }^{*}$ ，何大明 ${ }^{2}$, 李垣明 ${ }^{2}$, 陈平 ${ }^{2}$, 周毅 ${ }^{2}$

1. 复旦大学航空航天系, 力学与工程仿真研究所, 上海 200433;

2. 中国核动力研究设计院, 核反应堆系统设计技术重点实验室, 成都 610213

*联系人, E-mail: dingshurong@fudan.edu.cn

收稿日期: 2018-08-23; 接受日期: 2018-11-21; 网络出版日期: 2019-06-17

国家自然科学基金(编号: 11572091, 11772095)、国家重点研发计划(编号: 2016YFB0700103)和中国核动力研究设计院核反应堆系统设计技术 重点实验室项目资助

摘要在中子辐照的环境中, 由于裂变气体产物的产生, UMo燃料芯体将演化为多孔结构, 孔隙率和孔压随着燃 耗增长而不断变化，促使单片式燃料元件内产生复杂的多尺度辐照热力耦合行为. 本文针对 $\mathrm{UMo} / \mathrm{Al}$ 单片式燃料 元件, 基于考虑芯体亚晶化及外界静水压力相关性的裂变气体肿胀模型, 建立了芯体孔隙率随燃耗演化的理论模 型，进一步考虑孔隙率及孔压影响建立了芯体/包壳界面微观正应力计算模型. 将芯体孔隙率演化模型引入燃料 元件多尺度辐照热力耦合行为的三维有限元模拟, 实现了温度和孔隙率与芯体热传导率的实时关联, 获得了辐照 过程中燃料元件内热-力学场量的分布及演化规律, 计算分析了孔隙率对燃料元件温度、变形、芯体/包壳界面微 观正应力所产生的影响, 并考察了燃料元件表面热交换系数对其辐照热-力耦合行为的影响, 获得了芯体/包壳界 面破坏的重要影响因素.

关键词单片式燃料元件, 中子辐照, 裂变气体肿胀, 辐照蠕变, 多孔结构, 界面破坏机理

PACS: $24.75 .+\mathrm{i}, 44.10 .+\mathrm{i}, 46.15 .-\mathrm{x}$

\section{1 引言}

为了达到较高的中子通量, 高性能研究试验堆通 常采用高富集度铀燃料(HEU)，这埋下了核扩散的隐 患 ${ }^{[1]}$. 因此, 研究试验堆燃料低浓化计划(The Reduced Enrichment for Research and Test Reactors, RERTR)提 出将高富集度铀燃料替换为低富集度铀燃料(LEU ${ }^{[1-3]}$
的要求. 为了保证研究试验堆的高中子注量率, 需要提 高燃料的铀密度 ${ }^{[3]}$. UMo 合金燃料具有等效铀密度 高、辐照性能稳定等特点 ${ }^{[3-6]}$, 在研究试验堆中具有良 好的发展前景 ${ }^{[7,8]}$.

$\mathrm{UMo} / \mathrm{A} 1$ 单片式燃料元件由 UMo 合金芯体和 A16061包壳构成，芯体和包壳通过热等静压加工实现 冶金结合 ${ }^{[9]}$. 在反应堆苛刻的服役条件下，UMo/Al燃

引用格式: 严峰, 孔祥喆, 丁淑蓉, 等. 芯体多孔结构对单片式燃料元件辐照热-力耦合行为的影响研究. 中国科学: 物理学 力学 天文学, 2019, 49: 114606 Yan F, Kong X Z, Ding S R, et al. Effects of porous fuel structure on the irradiation-induced thermo-mechanical coupling behavior in monolithic fuel plates (in Chinese). Sci Sin-Phys Mech Astron, 2019, 49: 114606, doi: 10.1360/SSPMA2018-00297 
料元件会产生复杂的热力耦合行为. UMo合金因核裂 变释放热量、产生固体和气体裂变产物, 并产生辐照 蠕变; 随着燃耗的发展, 裂变产物逐渐增多 ${ }^{[8,9]}$, 使燃料 芯体产生逐渐增大的辐照肿胀 ${ }^{[9]}$; 芯体的辐照肿胀会 引起芯体与包壳间强烈的力学相互作用, 使燃料元件 产生大变形, 并引起包壳的塑性变形.

燃料芯体的辐照肿胀包括裂变固体肿胀和气体肿 胀, 其中裂变固体肿胀与燃耗成正比 ${ }^{[8]}$, 而裂变气体肿 胀的物理机制较为复杂，需考虑晶粒亚晶化效应和外 界静水压力的影响 ${ }^{[10]}$. 裂变气体在燃料芯体中形成晶 内及晶界气泡，使芯体产生受内压作用的孔洞。随着 燃耗的增长，芯体孔隙率逐渐增大，使燃料芯体演化 为多孔结构, 从而导致芯体热传导率 ${ }^{[11]}$ 和结构强度的 降低，使得单片式核燃料元件内产生复杂的热力耦合 行为. 图1展示了 $\mathrm{UMo} / \mathrm{Al}$ 单片式燃料元件辐照后的金 相图 ${ }^{[12]}$, 其芯体呈多孔结构, 在芯体和包壳的界面处 发生了开裂. 这一开裂行为会威胁元件的使用寿命和 服役安全性 ${ }^{[13]}$, 其与芯体的多孔结构及孔压密切相关. 为了预测 $\mathrm{UMo} / \mathrm{Al}$ 单片式核燃料元件内的辐照热-力耦 合行为, 分析芯体与包壳界面开裂的机理, 需要考虑孔 隙率的影响建立相关理论模型及数值模拟方法.

对于 $\mathrm{UMo} / \mathrm{Al}$ 燃料元件辐照热力耦合行为的数值 模拟研究, 已取得一些研究成果. Kim等人 ${ }^{[9]}$ 基于UMo 燃料的辐照肿胀经验公式 ${ }^{[8]}$, 利用二维有限元模型对 单片式燃料元件的堆内热力耦合行为进行了计算，考 察了UMo合金蠕变系数对元件厚度变化的影响.Cui 等人 ${ }^{[14]}$ 综合考虑晶界气体原子的重溶效应和外界静 水压力的影响, 得到计算UMo燃料裂变气体肿胀的半 解析解, 并引入 $\mathrm{UMo} / \mathrm{Al}$ 单片式燃料元件辐照热力耦合

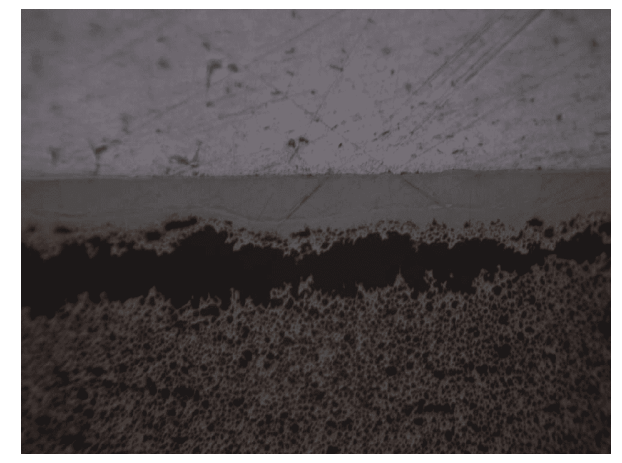

图 1 (网络版彩图)板L1P754的金相图 ${ }^{[12]}$

Figure 1 (Color online) Optical metallography image of fuel plate L1P04A [12].
行为的三维有限元模拟中. 上述研究将UMo燃料芯体 视为均质材料, 未考虑多孔结构对UMo燃料芯体热-力 学性能的影响, 也未研究芯体孔隙率对 $\mathrm{UMo} / \mathrm{Al}$ 燃料元 件的界面微观应力的影响. 目前, 界面开裂的机理尚未 得到合理的解释. 因此，需要结合UMo合金的裂变气 体肿胀模型，建立孔隙率随燃耗演化的理论模型，深 入考察多孔结构对单片式燃料元件辐照热力耦合行为 的影响, 探究燃料元件界面破坏的机理.

本文针对 $\mathrm{UMo} / \mathrm{Al}$ 单片式燃料元件, 建立了三维有 限元模型, 并在已有研究的基础上, 建立了孔隙率随燃 耗演化的理论模型以及芯体/包壳界面微观正应力的 计算模型. 综合考虑芯体辐照肿胀、辐照蠕变和包壳 热弹塑性变形的影响, 引入芯体孔隙率演化的理论模 型, 实现了元件多尺度辐照热力耦合行为的数值模拟, 获得了燃料元件辐照热力耦合行为的演化规律, 并探 究了芯体孔隙率所产生的影响, 解释了芯体与包壳界 面破坏的可能机理.

\section{2 辐照工况条件}

单片式燃料元件所处于的辐照工况条件决定于燃 料元件在反应堆中的放置方式 ${ }^{[9]}$. 在不同的中子辐照 工况条件下，燃料芯体的裂变率也会不同. 本文采用 了沿芯体宽度方向非均匀分布的裂变率，通过对实验 数据 ${ }^{[9]}$ 进行拟合, 可以得到裂变率的表达式为

$$
\begin{aligned}
\dot{f}= & 2.140 \times 10^{16} \bar{y}^{4}-1.003 \times 10^{18} \bar{y}^{3} \\
& +1.82 \times 10^{19} \bar{y}^{2} \\
& -1.651 \times 10^{20} \bar{y}+1.1398 \times 10^{21},
\end{aligned}
$$

其中, $\dot{f}$ 为裂变率, 单位是 fissions $\mathrm{m}^{-3} \mathrm{~s}^{-1}$ (下文简写为 $\mathrm{f}$ $\left.\mathrm{m}^{-3} \mathrm{~s}^{-1}\right) ; \bar{y}(\mathrm{~mm})$ 是芯体内的点距离裂变率最高一侧的 距离. 非均匀分布的裂变率见图2.

燃料芯体的产热率 $\dot{q}$ 跟裂变率 $\dot{f}$ 线性相关 ${ }^{[15]}$ :

$\dot{q}=c \cdot \dot{f}$,

其中, $c=3.204 \times 10^{-11} \mathrm{~J}$ 为单次裂变所释放的能量; 产热 率 $\dot{q}$ 的单位是 $\mathrm{W} / \mathrm{m}^{3}$.

\section{3 辐照环境下的材料热-力学性能模型}

\subsection{UMo合金}

(1) 热传导率 


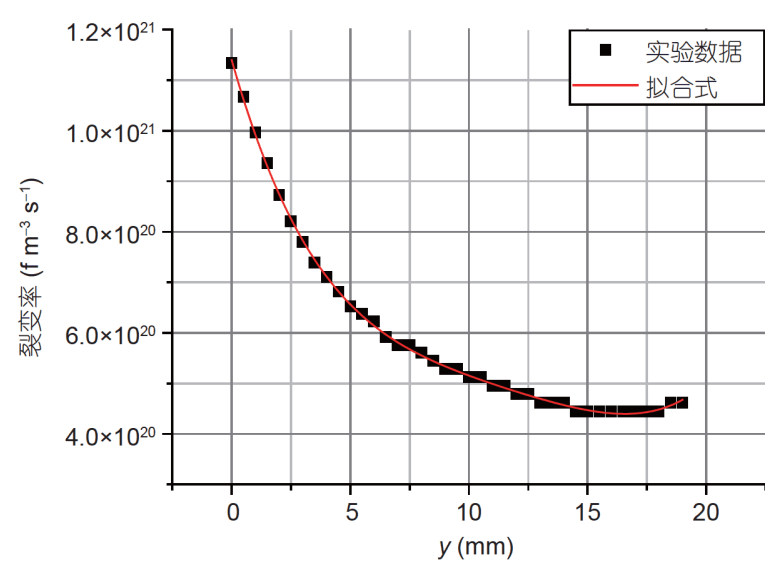

图 2 (网络版彩图)芯体内非均匀分布的裂变率

Figure 2 (Color online) Non-uniform fission rate distribution in the fuel meat.

$\mathrm{UMo}$ 合金的热传导率与燃耗和温度相关 ${ }^{[16]}$. 对于 未辐照的UMo合金, 其热传导率取决于Mo的质量百分 数 ${ }^{[16]}$ :

$$
\begin{aligned}
k_{\mathrm{UMo}}^{0}= & \left(1-\sqrt{1-\chi_{\mathrm{Mo}}}\right) k_{\mathrm{Mo}} \\
& +\sqrt{1-\chi_{\mathrm{Mo}}}\left\{\left(1-\chi_{\mathrm{Mo}}\right) k_{\mathrm{U}}+\chi_{\mathrm{Mo}} k_{\mathrm{c}, \mathrm{Mo}}\right\},
\end{aligned}
$$

式中, $k_{\mathrm{UMo}}^{0}$ 是未经辐照的UMo合金的热传导率, 单位是 $\mathrm{W} \mathrm{m}{ }^{-1} \mathrm{~K}^{-1} ; \chi_{\mathrm{Mo}}$ 是 $\mathrm{Mo}$ 的质量百分数, 本文采用 $10 \%$; $k_{\mathrm{Mo}}$ 和 $k_{\mathrm{U}}$ 依次是 $\mathrm{Mo}$ 和 $\mathrm{U}$ 的热传导率, $k_{\mathrm{c}, \mathrm{Mo}}$ 为修正项, 其 表达式如下:

$$
\begin{aligned}
k_{\mathrm{U}}(T)= & 21.73+1.591 \times 10^{-2} T \\
& +5.907 \times 10^{-6} T^{2}, \\
k_{\mathrm{Mo}}(T)= & 150.0-4.0 \times 10^{-2} T, \\
k_{\mathrm{c}, \mathrm{Mo}}= & -274.4+985.2 \chi_{\mathrm{Mo}}-1.941 \times 10^{3} \chi_{\mathrm{Mo}}^{2} \\
& +3.640 \times 10^{-2} T \\
& +7.365 \times 10^{-5} T^{2}+5.793 \times 10^{-2} \chi_{\mathrm{Mo}} T,
\end{aligned}
$$

其中, $k_{\mathrm{Mo}}, k_{\mathrm{U}}$ 和 $k_{\mathrm{c}, \mathrm{Mo}}$ 的单位均为 $\mathrm{W} \mathrm{m} \mathrm{m}^{-1} \mathrm{~K}^{-1}$; 温度 $T$ 的单 位为 $\mathrm{K}$.

辐照过程中, UMo合金会产生裂变气体, 形成气 孔, 其热传导率可以通过两相模型描述 ${ }^{[11]}$ :

$k_{\mathrm{UMo}}=0.25\left[A+\sqrt{A^{2}+8 k_{\mathrm{UMo}}^{0} k_{\mathrm{g}}}\right]$,

其中, $k_{\mathrm{g}}$ 表示气孔的热传导率, 单位 $\mathrm{W} \mathrm{m}^{-1} \mathrm{~K}^{-1} ; k_{\mathrm{g}}$ 和 $A$ 的表达式如下:

$$
\begin{aligned}
k_{\mathrm{g}}= & 0.1\left(8.247 \times 10^{-5} T^{0.8363}\right) \\
& +0.9\left(4.351 \times 10^{-5} T^{0.8616}\right), \\
A= & (2-3 \text { Poro }) k_{\mathrm{UMo}}^{0}+(3 \text { Poro }-1) k_{\mathrm{g}},
\end{aligned}
$$

其中, 温度 $T$ 的单位为 $\mathrm{K}$; Poro表示芯体孔隙率, 具体计 算方法见3.2节.

(2) 热膨胀系数

$\mathrm{UMo}$ 合金的热膨胀系数 $\alpha\left(\mathrm{K}^{-1}\right)$ 与温度 $T(\mathrm{~K})$ 相 关 $^{[15,17]}$ :

$\alpha=\left\{\begin{array}{c}3.0 \times 10^{-11} T^{2}-1.698 \times 10^{-8} T \\ +1.395 \times 10^{-5}, T \leq 673 \mathrm{~K} \\ -5.0 \times 10^{-12} T^{2}+1.023 \times 10^{-8} T \\ +1.148 \times 10^{-5}, T \geq 673 \mathrm{~K} .\end{array}\right.$

(3) 辐照蠕变

等效蠕变应变率与等效应力和裂变率线性相

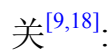

$\dot{\varepsilon}_{\mathrm{c}}=B \sigma \dot{f}$,

其中, $\dot{\varepsilon}_{\mathrm{c}}$ 为等效蠕变应变率, $\mathrm{s}^{-1} ; B=5 \times 10^{-35} \mathrm{~m}^{3} / \mathrm{Pa}$ 为蠕 变率系数; $\sigma$ 为等效应力, $\mathrm{Pa} ; \dot{f}$ 为裂变率, $\mathrm{f} \mathrm{m}^{-3} \mathrm{~s}^{-1}$.

(4) 辐照肿胀

$\mathrm{UMo}$ 合金的辐照肿胀包括裂变固体肿胀和裂变 气体肿胀, 其中裂变固体肿胀与裂变密度线性相 关 $^{[9,19]}$ :

$\frac{\Delta V_{\text {solid }}}{V_{0}}=4.0 f_{\mathrm{d}}$,

式中, $f_{\mathrm{d}}$ 为裂变密度, 单位为 $10^{27}$ fissions $/ \mathrm{m}^{3}$ (下文简写 为 $\left.\mathrm{f} / \mathrm{m}^{3}\right)$.

裂变气体肿胀的物理机制较为复杂, 需要考虑晶 界重溶和亚晶化效应 ${ }^{[14,20,21]}$. 根据亚晶化理论, 当燃耗 达到拐点燃耗之后, 原始晶粒由边界向内部亚晶化. 拐 点燃耗 $F_{\mathrm{dx}}$ 可以表示为 ${ }^{[14]}$

$F_{\mathrm{dx}}=6 \times 10^{24}(\dot{f})^{\frac{2}{15}} \mathrm{f} / \mathrm{m}^{3}$,

其中, 裂变率 $\dot{f}$ 的单位是 $\mathrm{f}^{-3} \mathrm{~s}^{-1}$.

若燃料末达到拐点燃耗, 裂变气体肿胀主要由晶 内气泡肿胀和晶界气泡肿胀引起, 即 ${ }^{[14,21]}$ :

$\frac{\Delta V_{\text {gas }}}{V_{0}}=\left.\left(\frac{\Delta V_{\text {intra }}}{V_{0}}\right)\right|_{r_{\mathrm{gr}}}+\left.\left(\frac{\Delta V_{\text {inter }}}{V_{0}}\right)\right|_{r_{\mathrm{gr}}}$, 
其中, $\left.\left(\frac{\Delta V_{\text {intra }}}{V_{0}}\right)\right|_{r_{\mathrm{gr}}}$ 为晶内气泡肿胀; $\left.\left(\frac{\Delta V_{\text {inter }}}{V_{0}}\right)\right|_{r_{\mathrm{gr}}}$ 为晶界气 泡肿胀, $r_{\mathrm{gr}}$ 为原始晶粒的半径.

亚晶化之后，晶粒分为亚晶化区域和未亚晶化区 域，其中亚晶化区域的裂变气体肿胀主要由晶界气泡 肿胀导致 ${ }^{[21]}$. 燃料芯体的裂变气体肿胀可表示为 ${ }^{[14]}$

$$
\begin{aligned}
\frac{\Delta V_{\text {gas }}}{V_{0}}= & \left(1-V_{r}\right)\left[\left.\left(\frac{\Delta V_{\text {intra }}}{V_{0}}\right)\right|_{r_{\mathrm{gr}}}+\left.\left(\frac{\Delta V_{\text {inter }}}{V_{0}}\right)\right|_{r_{\mathrm{gr}}}\right] \\
& +\left.V_{r}\left(\frac{\Delta V_{\text {inter }}}{V_{0}}\right)\right|_{r_{\mathrm{grx}}},
\end{aligned}
$$

其中, $V_{r}$ 为原始晶粒内亚晶化区域的体积分数; $\left.\left(\frac{\Delta V_{\text {inter }}}{V_{0}}\right)\right|_{r_{\mathrm{grx}}}$ 为亚晶化区域的晶界气泡肿胀; $r_{\mathrm{rgx}}$ 为亚晶 化区域的晶粒半径.

对于晶界气泡的压强和半径, 需满足修正的范德 华方程:

$$
\left(\frac{2 \gamma}{R_{\mathrm{b}}}+p\right)\left(\frac{4 \pi R_{\mathrm{b}}^{3}}{3}-h_{\mathrm{s}} b_{\mathrm{v}} N_{\mathrm{b}}\right)=N_{\mathrm{b}} k T,
$$

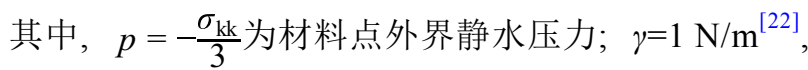
$2 \gamma / R_{\mathrm{b}}$ 为气泡表面张力, 单位是 $\mathrm{Pa} ; R_{\mathrm{b}}$ 为晶界气泡半径, $\mathrm{m} ; 2 \gamma / R_{\mathrm{b}}+p$ 为晶界气泡内压; $h_{\mathrm{s}}=0.6$ 为无量纲常数 ${ }^{[23]}$; $b_{\mathrm{v}}=8.5 \times 10^{-29} \mathrm{~m}^{3}$ 为Xe的范德华常数 ${ }^{[22]} ; N_{\mathrm{b}}=N / C_{\mathrm{b}}$ 为每个 晶界气泡内的平均气体原子数; $k=1.38 \times 10^{-23} \mathrm{~J} / \mathrm{K}$ 为玻 尔兹曼常数.

晶粒尺度上的裂变气体扩散行为与核燃料的裂变 气体肿胀相关联. 关于UMo合金裂变气体肿胀的详细 模型及参数见文献[14], 此处不再赘述.

(5) $\mathrm{UMo}$ 合金的杨氏模量为 $85 \mathrm{GPa}$; 泊松比为 $0.34^{[9]}$.

\subsection{Al6061合金}

(1) 热传导率

Al6061合金的热传导率 $k\left(\mathrm{~W} \mathrm{~m}^{-1} \mathrm{~K}^{-1}\right)$ 与温度 $T(\mathrm{~K})$ 相关 ${ }^{[17,24]}$ :

$k=-1.77 \times 10^{-4} T^{2}+0.19 T+138.55$.

(2) 热膨胀系数

对实验数据进行拟合 ${ }^{[17]}$, 可以得到热膨胀系数 $\alpha_{c}$
$\left(\mathrm{K}^{-1}\right)$ 与温度 $T(\mathrm{~K})$ 的关系式 ${ }^{[24]}$ :

$\alpha_{\mathrm{c}}=(0.9 T+2018) \times 10^{-8}$.

(3) 硬化曲线

$\mathrm{A} 16061$ 合金硬化阶段的应力 $\sigma(\mathrm{MPa})$-应变 $\varepsilon$ 曲 线为 ${ }^{[9]}$

$\sigma=K \varepsilon^{n}$,

其中, $K=569.6 \mathrm{MPa}$ 为强度系数; $n=0.13$ 为应变硬化 指数.

(4) Al6061的杨氏模量为 $66 \mathrm{GPa}$; 泊松比为 $0.34^{[9]}$.

\section{4 理论模型}

\section{1 应力更新算法}

为实现燃料元件堆内复杂热力耦合行为的有限元 模拟, 需结合燃料元件的材料力学性能模型, 建立旋转 坐标系下三维大变形增量本构关系, 并在通用有限元 软件ABAQUS的框架内编写了相应的UMAT子程序. 在每个增量步中, 考虑UMo合金材料点的总应变增量 包含弹性应变、热膨胀应变、辐照肿胀应变与辐照蠕 变应变增量的贡献, 其弹性应变增量 $\Delta \varepsilon_{i j}^{\mathrm{e}}$ 可表示为

$\Delta \varepsilon_{i j}^{\mathrm{e}}=\Delta \varepsilon_{i j}^{\mathrm{total}}-\Delta \varepsilon_{i j}^{\mathrm{th}}-\Delta \varepsilon_{i j}^{\mathrm{sw}}-\Delta \varepsilon_{i j}^{\mathrm{c}}$,

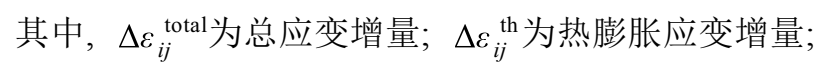
$\Delta \varepsilon_{i j}^{\mathrm{sw}}$ 为辐照肿胀应变增量; $\Delta \varepsilon_{i j}^{\mathrm{c}}$ 为辐照蠕变应变增量.

对于A16061包壳, 需考虑热膨胀应变与塑性应变 增量的影响, 即:

$\Delta \varepsilon_{i j}^{\mathrm{e}}=\Delta \varepsilon_{i j}^{\text {total }}-\Delta \varepsilon_{i j}^{\text {th }}-\Delta \varepsilon_{i j}^{\mathrm{p}}$,

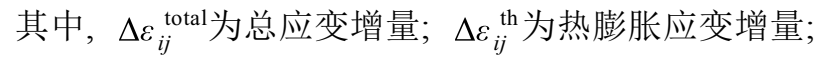
$\Delta \varepsilon_{i j}^{\mathrm{p}}$ 为塑性应变增量. 具体的应力更新算法参见文 献[25].

\section{2 孔隙率随燃耗的演化模型}

在辐照环境中, 燃料芯体裂变产生固体和气体裂 变产物 ${ }^{[8]}$. 随着燃耗的发展, 裂变气体在芯体中不断积 累, 形成如图1所示的小气孔，从而影响燃料芯体的热 传导率和力学性能. 因此, 需要结合裂变气体肿胀模 型, 建立孔隙率随燃耗演化的理论模型, 如下: 


$$
\begin{aligned}
\text { Poro } & =\frac{\Delta V_{\text {gas }}}{V}=\frac{\Delta V_{\text {gas }}}{V_{0}+\Delta V_{\text {gas }}+\Delta V_{\text {solid }}} \\
= & \frac{\frac{\Delta V_{\text {gas }}}{V_{0}}}{1+\frac{\Delta V_{\text {gas }}}{V_{0}}+\frac{\Delta V_{\text {solid }}}{V_{0}}},
\end{aligned}
$$

其中, $V_{0}$ 为原始体积; $V$ 为当前体积; $\frac{\Delta V_{\mathrm{gas}}}{V_{0}}$ 为裂变气体 肿胀, 本文所采用的裂变气体肿胀与温度和外界静水 压力相关, 并受到亚晶化效应的影响, 所以, 芯体的孔 隙率随时间和空间变化; $\frac{\Delta V_{\text {solid }}}{V_{0}}$ 为裂变固体肿胀, 其表 达式见式(12).

随时间及空间变化的孔隙率，致使芯体的热传导 率亦随时间和空间变化，使得元件内产生复杂的多尺 度辐照热力耦合行为. 本研究通过编制用户自定义子 程序，将材料点的热传导率与温度及孔隙率实时地关 联起来，实现了多尺度辐照热力耦合行为的三维数值 模拟.

\section{3 芯体/包壳界面微观正应力的计算模型}

在有限元模拟中, UMo合金芯体被视为均质材料. 但实际辐照过程中, 随着裂变气体的不断产生, 燃料芯 体内会形成许多小气孔, 成为多孔材料, 如图1所示. 对 于多孔材料，由于气孔受到内压作用，芯体/包壳界面 所受微观应力取决于孔隙率及孔压，与均匀化后的宏 观界面应力不同. 为了计算出界面微观正应力, 本研 究建立了界面微观正应力与宏观正应力之间的关系.

（1）为了简化模型，假设微观气孔在芯体/包壳界 面处以简单立方的形式排布, 选取如图3(a)所示的代

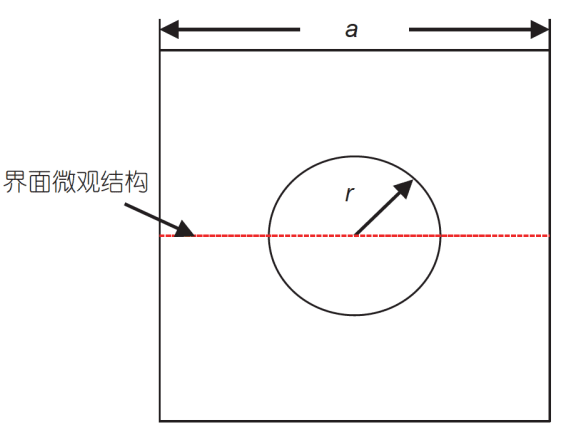

(a)
表性体元. 代表性体元的长宽高均为 $a$, 内部包含半径 为 $r$ 的等效气孔, $a$ 与 $r$ 需满足:

$\frac{\frac{4}{3} \pi r^{3}}{a^{3}}=$ Poro, 即 $\frac{r}{a}=\left(\frac{3 \cdot \text { Poro }}{4 \pi}\right)^{\frac{1}{3}}$.

（2）芯体的多孔结构使得界面的实际承载面积减 少, 致使界面微观正应力不同于宏观界面正应力, 如 图3(b)所示. 图中 $\sigma_{\mathrm{h}}$ 为均匀化的宏观界面正应力, $p_{\text {in }}$ 为 等效气孔所受的裂变气体压强, $\sigma_{\mathrm{g}}$ 为所求的界面微观 正应力. 根据平衡方程, 有:

$\sigma_{\mathrm{h}} a^{2}+p_{\text {in }} \pi r^{2}=\sigma_{\mathrm{g}}\left(a^{2}-\pi r^{2}\right)$.

整理可得界面微观正应力的表达式为

$$
\sigma_{\mathrm{g}}=\frac{\sigma_{\mathrm{h}}+p_{\text {in }} \pi\left(\frac{r}{a}\right)^{2}}{1-\pi\left(\frac{r}{a}\right)^{2}}=\frac{\sigma_{\mathrm{h}}+p_{\text {in }} \pi\left(\frac{3 \cdot \text { Poro }}{4 \pi}\right)^{\frac{2}{3}}}{1-\pi\left(\frac{3 \cdot \text { Poro }}{4 \pi}\right)^{\frac{2}{3}}} .
$$

为了探究芯体与包壳界面破坏的机理, 本文计算 分析芯体/包壳界面上的微观正应力的分布及演化规 律. 对于等效气孔的裂变气体压强 $p_{\mathrm{in}}$, 高利军等人 ${ }^{[26]}$ 提出 $\mathrm{UO}_{2}$ 燃料在高燃耗下裂变气体压力在 $100 \mathrm{MPa}$ 量 级, 本文暂时采用 $100 \mathrm{MPa}$ 的气孔内压来计算界面微 观正应力, 后续研究将进一步建立等效气孔内裂变气 体压强的理论模型. 这样, 通过有限元计算获得芯体/ 包壳界面宏观正应力之后，即可根据式(24)计算得到 界面微观正应力.

\section{5 有限元模型}

本文以RERTR-9A实验中编号为L1P04A的燃料

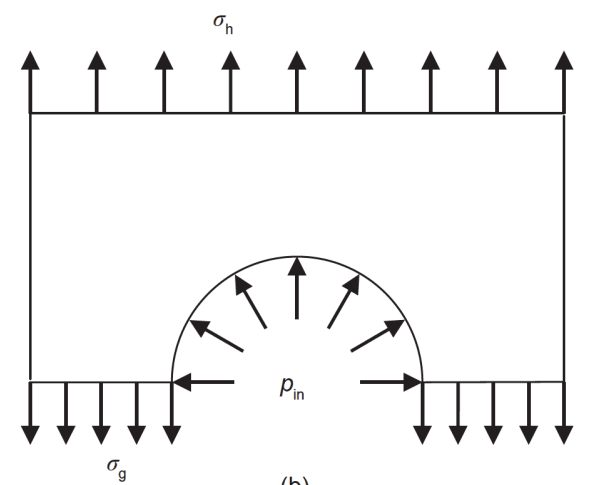

(b)

图 3 (网络版彩图)界面层微观结构(a)和界面微观正应力(b)示意图

Figure 3 (Color online) The diagrams of (a) interface microstructure and (b) microscopic interfacial normal stress. 
板为研究对象, 其包壳尺寸为 $100 \mathrm{~mm} \times 25 \mathrm{~mm} \times 1.4 \mathrm{~mm}$, 芯体尺寸为 $82.6 \mathrm{~mm} \times 19 \mathrm{~mm} \times 0.25 \mathrm{~mm}$ ，芯体位于燃料 板正中心 ${ }^{[9]}$. 为了提高计算效率, 考虑燃料板结构和辐 照条件的对称性, 取燃料板的 $1 / 4$ 建立三维有限元模型, 如图4(a)所示. 燃料板的两侧为固定边界; 下表面为热 交换边界, 取热交换系数 $h=0.035 \mathrm{~W} \mathrm{~mm}^{-2} \mathrm{~K}^{-1}$, 冷却剂 温度为 $323 \mathrm{~K}$; 其他边界条件如图4(a)所示. 在图4(b)中, 芯体的右侧所受中子辐照强度最高, 具有最大的裂变 率. 芯体内各点的裂变率随位置变化，分布规律见 式(1). 计算采用的单元数为 545370 , 已验证收玫性, 有 限元网格见图5.

\section{6 结果与讨论}

本文考虑核燃料及包壳的复杂辐照热-力学性能, 建立了模拟其多尺度辐照热力耦合行为的计算方法, 对元件内的热-力学行为进行了三维的有限元模拟. 本 节将分析燃料元件的辐照热-力学场量的分布及演化 规律, 并考察孔隙率所产生的影响.

\section{1 芯体孔隙率的分布和演化规律}

辐照环境中, 燃料芯体裂变产生的裂变气体产物 在芯体内聚集、形成孔洞，使芯体演化为多孔结构， 其孔隙率会影响燃料芯体的热-力学性能. 图6(a)展示 了辐照140天时芯体孔隙率的分布云图. 可以看出, 芯 体孔隙率总体沿 $y$ 轴方向逐渐降低, 与裂变率的分布规 律类似.从图6(a)中也可以看到，在路径 1 上部靠近燃 料板边缘处, 孔隙率呈现出与其他区域不同的分布规 律. 如3.2节所述, 芯体孔隙率的分布和演化规律与裂 变气体肿胀有关. 孔隙率的这种分布现象与芯体材料 裂变气体肿胀的非均匀分布有关. 图7(a)展示了辐照 140 天时裂变气体肿胀分布云图, 可以发现其与孔隙 率的分布云图类似, 在燃料板长度方向上的芯体/包壳 界面附近，燃料芯体的裂变气体肿胀出现了与其他区 域不同的分布规律, 这主要是因为裂变气体肿胀与温 度及外界静水压力相关.

图6(b) 展示了路径 1 上不同时刻芯体孔隙率的计 算结果. 可以看出, 随着辐照时间的增加, 芯体孔隙率 逐渐增大. 辐照 140 天时芯体孔隙率的最大值为 $24 \%$ 左 右. 可以发现, 在 100 天之前的辐照时间内, 孔隙率始终 在路径 1 的起始点最大. 但从 120 天及 140 天的计算结果 可以看出, 最大的孔隙率出现在离开起始点一定距离 的地方. 从式(22)可知, 这需要分析裂变固体肿胀及裂 变气体肿胀的分布及演化规律.

图7(b)给出了路径 1 上各点裂变气体肿胀随时间 的演化结果. 可以看出, 随着辐照时间的增加, 芯体裂 变密度逐渐增大, 裂变产生的气体产物逐渐增多, 裂变 气体肿胀亦逐渐增大. 辐照 140 天时裂变气体肿胀的最 大值高达 $44.9 \%$ 左右. 另外, 可以看出, 在 120 天的辐照 时间之前, 裂变气体肿胀总体上均沿路径 1 方向逐渐降 低, 这是因为裂变气体肿胀与燃料裂变率正相关, 两者 沿宽度方向变化趋势相似. 但从辐照 140 天时的裂变气

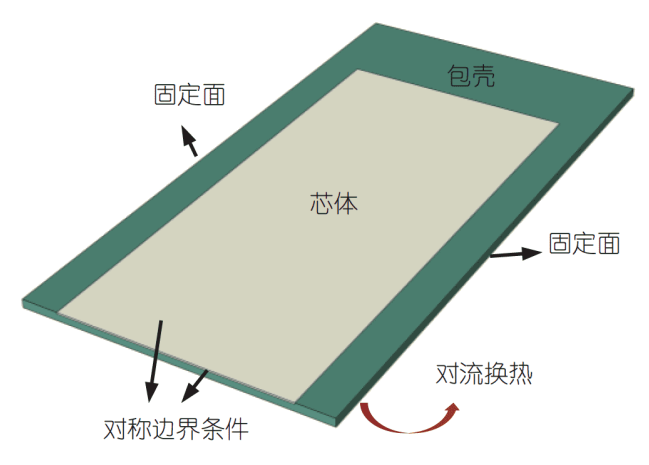

(a)

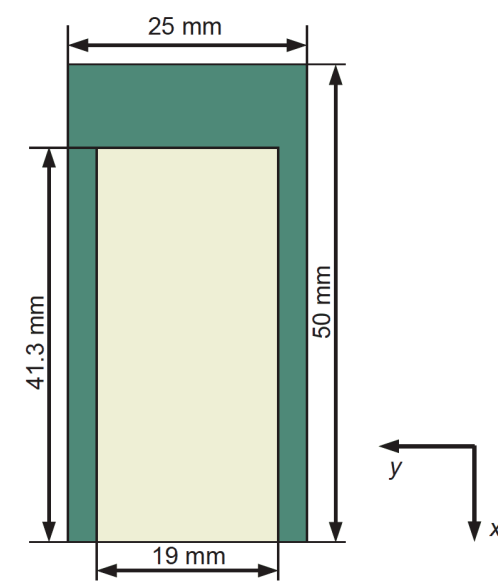

(b)

图 4 (网络版彩图)有限元几何模型示意图(a)和有限元模型的结构尺寸(b)

Figure 4 (Color online) The sketch of finite element model (a) and dimensions of finite element model (b). 


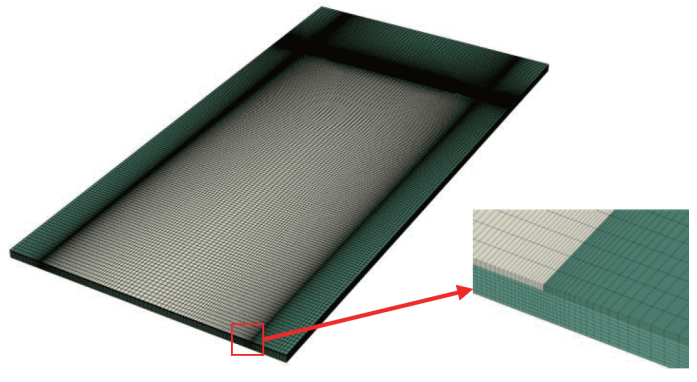

图 5 (网络版彩图)有限元网格图

Figure 5 (Color online) Finite element mesh grids.

体肿胀结果看到, 在离开路径起始点一定距离的位置 出现了一个极值点，在起始点与极值点之间出现了一 个较低的值. 从图 2 可知, 裂变密度沿着路径 1 单调降
低， 140 天时的裂变气体肿胀分布特征主要与静水压 力和温度相关, 下面将分析温度和静水压力对裂变气 体肿胀的影响. 将图6(b)及图7(b)140天时的结果进行 对比可以发现, 裂变气体肿胀最大处的孔隙率不是最 高的, 这要从式(22)分析原因. 由式(12)可知, 裂变固 体肿胀与裂变密度成正比, 裂变率最高的点, 即路径 1 起始点的裂变固体肿胀最大, 所以, 在裂变气体肿胀相 差不大的临近点, 其孔隙率反而会大于起始点的数值.

本文采用的裂变气体肿胀模型考虑了外界静水压 力的影响, 裂变气体肿胀的计算结果也反映了静水压 力相关性. 图8(a)给出了辐照140天后、由路径 1 和路 径 2 输出的裂变气体肿胀结果. 路径 2 上各点的裂变气 体肿胀低于路径 1 的数值，相对差别在 $6.7 \%-30.8 \%$ 范

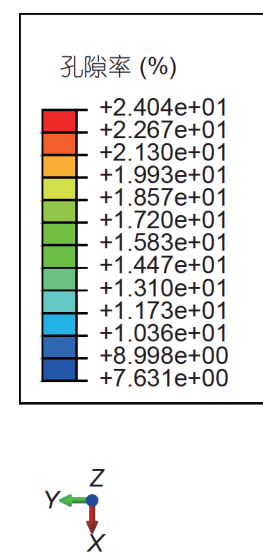

(a)

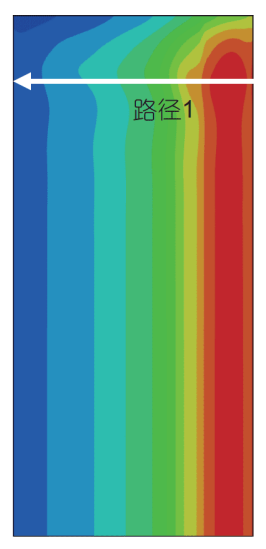

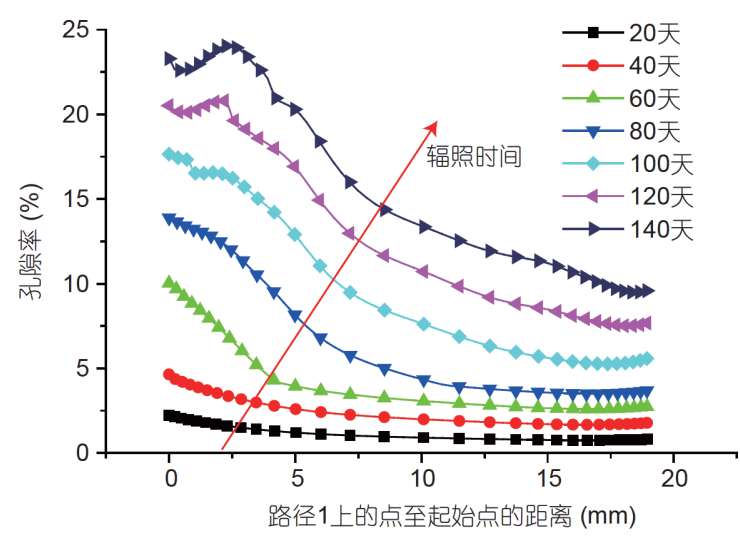

(b)

图 6 (网络版彩图)燃料芯体孔隙率分布云图(a)和孔隙率随辐照时间沿路径1的演化结果(b)

Figure 6 (Color online) The contour plot of fuel porosity (a) and the evolution rules of porosity along Path 1 (b).

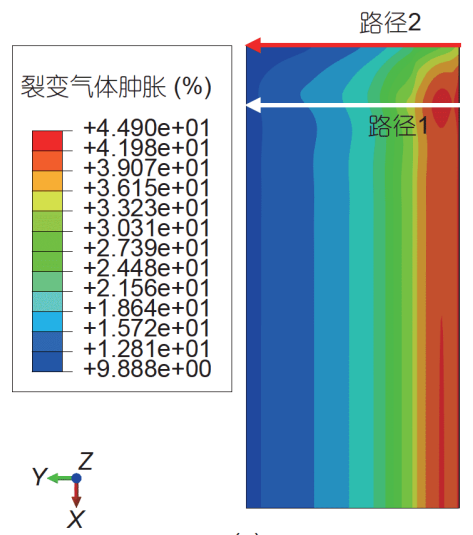

(a)

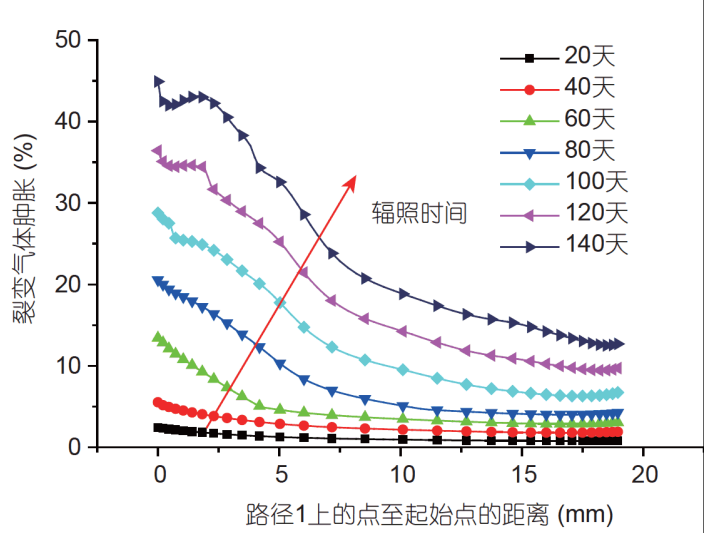

(b)

图 7 (网络版彩图)裂变气体肿胀分布云图(a)和裂变气体肿胀随辐照时间沿路径1的演化结果(b)

Figure 7 (Color online) The contour plot of fission gas swelling (a) and the evolution rules of fission gas swelling along Path 1 (b). 


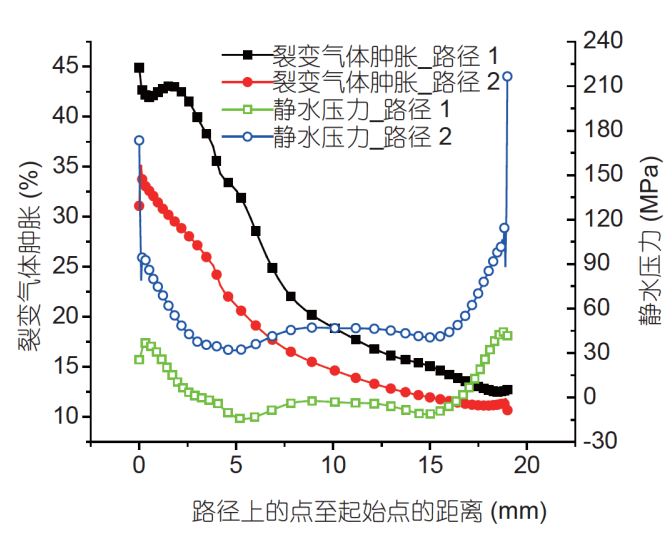

(a)

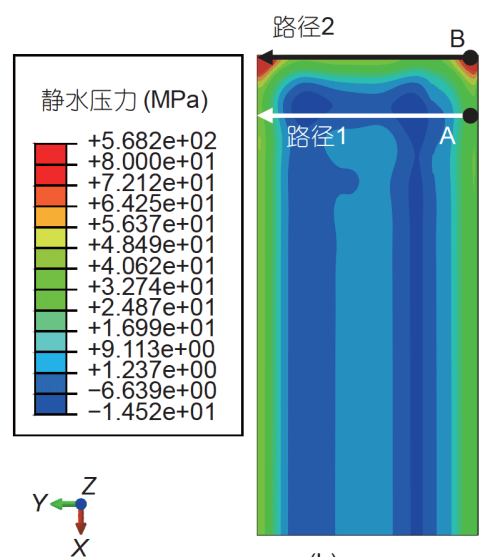

(b)

图 8 (网络版彩图)不同路径的静水压力和裂变气体肿胀(a)以及芯体静水压力分布云图(b)

Figure 8 (Color online) Hydrostatic pressure and fission gas swelling along different paths (a) and the contour plot of hydrostatic pressure (b).

围之内. 由式(16)可知, 这一方面因为路径2靠近包壳, 温度较低. 另一个很重要的原因是来自于外界静水压 力的影响. 图8(b)展示了燃料芯体静水压力的分布云 图. 可以看出, 路径 1 和 2 处的静水压力产生了很大的 差别. 在图8(a)中, 同时给出了两条路径上的静水压力 分布曲线, 可以看到路径 2 的起始点处有很大的静水压 力, 这可能是导致路径 2 起始点的裂变气体肿胀明显低 于邻近点的主要原因. 路径 2 的起始点位于芯体的角 部，在长度和宽度方向的变形都受到包壳的约束，当 芯体产生辐照肿胀时，此处的芯体和包壳产生了强烈 的相互挤压的作用, 所以, 产生了很大的静水压力, 使 得局部的裂变气体肿胀数值减小.

不同于辐照肿胀的经验公式 ${ }^{[8]}$, 本文的裂变气体 肿胀模型同时考虑了静水压力和温度的相关性. 为了 细致地分析温度和静水压力对裂变气体肿胀的影响, 选取了路径 1 和路径 2 的起始点 (记为 $\mathrm{A}$ 点和 $\mathrm{B}$ 点) 的晶 界气泡半径进行了分析, 重点考虑了温度和静水压力 对晶界气泡半径的影响, 具体见表1. 从表1可以看到: 若 $\mathrm{A}$ 点温度下降至 $364.3 \mathrm{~K}$, 即 $\mathrm{B}$ 点温度, 根据式(16)所 计算出的晶界气泡半径将减小 $2.1 \%$ 左右; 若 $\mathrm{A}$ 点温度 保持为 $410.5 \mathrm{~K}$, 静水压力升高到 $173.4 \mathrm{MPa}$, 所计算出 的晶界气泡半径将减小约 $12.5 \%$. 考虑到裂变气体肿 胀与晶界气泡半径的三次方近似成正比, $\mathrm{A}$ 点裂变气 体肿胀将减小 $33 \%$ 左右，下降幅度非常明显. 由此可 见, 静水压力对裂变气体辐照肿胀有着较为显著的影 响, $\mathrm{B}$ 点处有很大的静水压力是 $\mathrm{B}$ 点裂变气体肿胀远低 于 $A$ 点的主要原因.
表 1 温度和静水压力对晶界气泡半径的影响

Table 1 Effects of temperature and hydrostatic pressure on the radius of intergranular bubbles

\begin{tabular}{ccccc}
\hline 参数 & A 点 & $\mathrm{B}$ 点 & $\mathrm{A}^{\prime}$ & $\mathrm{A}^{\prime \prime}$ \\
\hline $\begin{array}{c}\text { 温度 }(\mathrm{K}) \\
\text { 静水压力 } \\
(\mathrm{MPa})\end{array}$ & 410.5 & 364.3 & 364.3 & 410.5 \\
$\begin{array}{c}\text { 晶界气泡 } \\
\text { 半径 }(\mathrm{mm})\end{array}$ & $2.79 \times 10^{-5}$ & $2.40 \times 10^{-5}$ & $2.73 \times 10^{-5}$ & $2.44 \times 10^{-5}$ \\
\hline
\end{tabular}

可见, 主要是因为裂变气体肿胀的温度及静水压 力相关性，使得芯体的孔隙率产生了图6所示的分布 及演化规律.

在本课题组早期的研究中 ${ }^{[27]}$, 曾采用Kim等人 ${ }^{[8]}$ 提出的裂变气体肿胀经验模型. 该模型没有考虑温度 与静水压力的影响, 其所计算出的芯体孔隙率及裂变 气体肿胀见图9(a)和(b). 可以看出其与图6(a)与7(a)不 同, 由经验公式计算得到的孔隙率和裂变气体肿胀沿 $x$ 轴方向几乎没有变化, 沿 $y$ 轴方向逐渐降低, 最大值出 现在裂变率最大的一侧. 由于没有考虑裂变气体肿胀 的静水压力相关性, 所计算出的孔隙率及裂变气体肿 胀明显偏高于考虑静水压力相关性的计算结果.

\section{2 燃料元件的变形}

辐照过程中, 燃料芯体裂变产生的固体和气体裂 变产物不断积累, 引起较大的辐照肿胀, 从而使燃料 元件产生较大的变形, 芯体与包壳之间产生强烈的力 学相互作用. 由于燃料元件的两侧被固定, 元件的变 


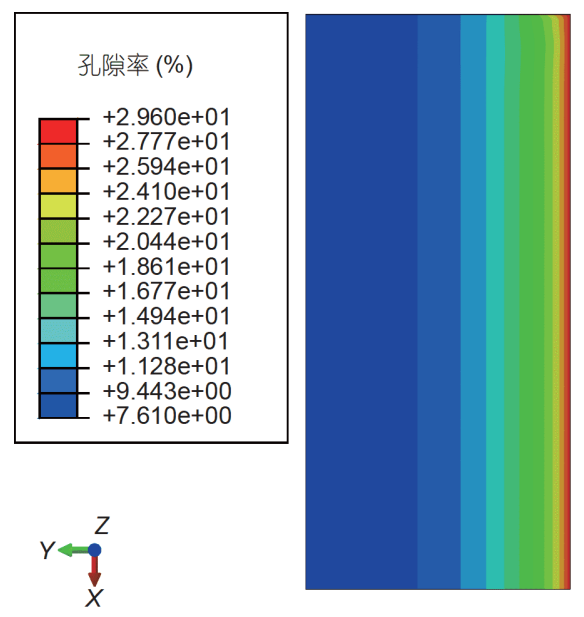

(a)

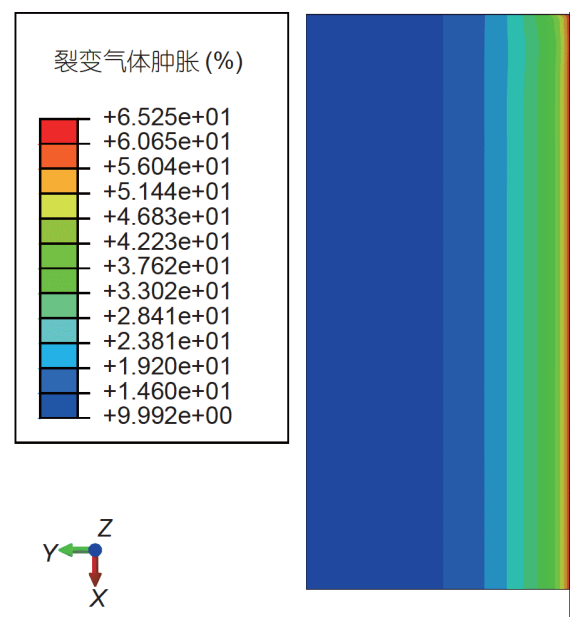

(b)

图 9 (网络版彩图)辐照 140 天时的芯体孔隙率分布云图(a)和裂变气体肿胀云图(b)

Figure 9 (Color online) The contour plots of fuel porosity (a) and fission gas swelling (b) on the 140th day.

形主要体现为厚度变化 ${ }^{[9]}$. 在产生辐照肿胀的同时, 芯 体还产生辐照蠕变, 它们都会对芯体的变形产生重要 的贡献.

图10(a)给出了燃料元件在辐照 140 天时的相对厚 度增量云图. 图中展示的平面为燃料元件的外表面, 即 图4(a)所示模型的下表面. 芯体的裂变率沿图中 $y$ 轴方 向逐渐降低. 从图10(a)可以看出，燃料元件边缘处包 壳部分的厚度增量基本为零，而下面包裹着芯体的包 壳区域在芯体的挤压下产生了较大的厚度增量, 其中 厚度增量的最大值出现在靠近辐照强度最高的一侧、 位于芯体角落附近. 图10(b)展示了燃料元件的相对厚 度增量随时间的演化规律. 可以看出, 随着辐照时间的
增加, 元件的相对厚度增量逐渐升高. 辐照 140 天时燃 料元件的绝对厚度增量的最大值为 $0.155 \mathrm{~mm}$, 相对于 初始厚度增长约 $22.12 \%$.

对比图10(b)和2可以看出：燃料元件相对厚度增 量的最大值出现在辐照最强、芯体裂变率最大边缘的 附近, 与芯体与包壳界面存在一段距离, 而靠近芯体与 包壳界面处的相对厚度增量接近于零. 这一现象与 RERTR 实验的观测结果相符 ${ }^{[9]}$. 在芯体辐照最强的一 侧, 芯体的辐照肿胀很大, 但此处元件的厚度变化很 小，这与芯体的辐照蠕变变形密切相关. 图11(a)给出 了辐照 140 天时芯体辐照肿胀应变和辐照蠕变应变在 厚度方向的分量，其中路径1的位置见图6(a). 可以看

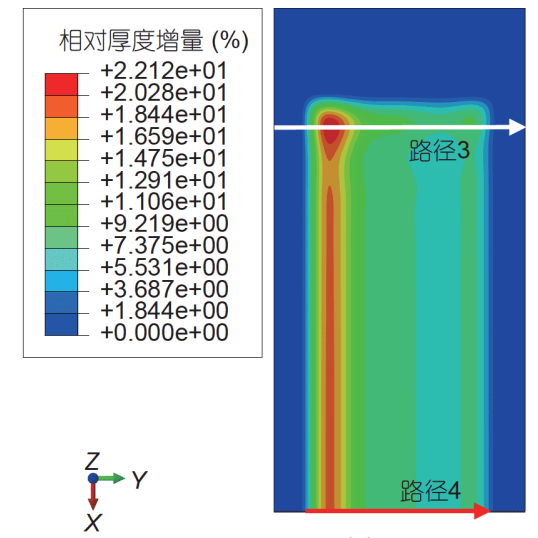

(a)

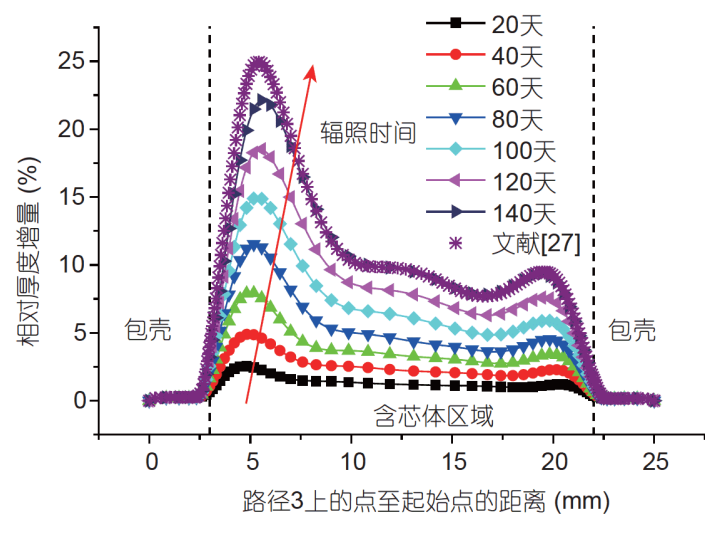

(b)

图 10 (网络版彩图)相对厚度增量分布云图(a)和相对厚度增量随辐照时间沿路径3的演化结果(b)

Figure 10 (Color online) The contour plot of relative thickness increment (a) and its evolution results along Path 3 (b). 

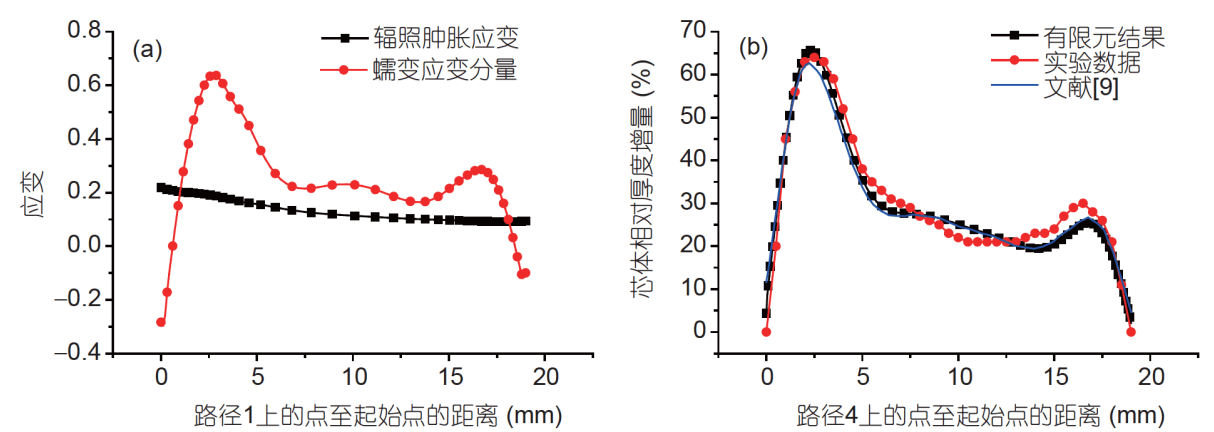

图 11 (网络版彩图) (a) 辐照 140 天时辐照肿胀应变和辐照蠕变应变分量沿路径 1 的分布曲线; (b) 辐照98天时本研究的有限元 结果与文献[9]中的实验数据和数值模拟结果的对比

Figure 11 (Color online) (a) The distribution curves of irradiation swelling strain and irradiation creep strain component along Path 1 on the 140th day and (b) the comparisons of FEM result in this work with experimental data and stimulation result from ref. [9] on the 98th day irradiation.

出, 总的辐照肿胀应变(包括裂变固体肿胀和裂变气体 肿胀) 沿路径 1 的方向逐渐降低, 在辐照最强的一侧数 值最大. 辐照蠕变增量的分布规律比较复杂, 分布曲 线的形状与元件相对厚度增量曲线类似, 这说明元件 相对厚度增量的分布规律主要受到辐照蠕变的影响. 另外, 可以看到, 在路径的两端, 辐照蠕变应变分量为 负数, 与辐照肿胀应变分量相叠加几乎为零, 所以, 在 辐照蠕变和辐照肿胀的共同作用下, 在芯体与包壳的 界面附近元件的相对厚度增量几乎为零.

图10(b)对比了辐照140天时本研究和文献[27]所 得元件相对厚度增量的计算结果. 可以看出, 采用 Kim 等人 ${ }^{[8]}$ 提出的裂变气体肿胀经验公式, 所得到的燃料 元件相对厚度增量的峰值比本研究大 $2.82 \%$ 左右, 路 径上其他点的计算结果很相近. 在Kim等人 ${ }^{[9]}$ 的论文 中提供了板L1P04A在辐照 98 天时芯体相对厚度增量
的实验数据 ${ }^{[9]}$, 并给出了采用裂变气体肿胀经验公式 所得到的数值模拟结果. 图11(b)比较了本研究的有限 元结果、实验数据以及 Kim的数值模拟结果的差异. 可以看出两种裂变气体肿胀模型下的计算结果非常相 近. 可以发现本文的计算结果与实验数据差距更小, 特 别是在路径的两端点处，这验证了本文所采用的裂变 气体肿胀模型及所建立数值模拟方法的正确性.

正如前面所提到的，由于元件的厚度变化很大程 度上受到辐照蠕变的影响, 所以, 基于两种裂变气体 肿胀模型所算出的元件厚度增量差别不大.

\section{3 燃料元件温度场的分布和演化}

辐照98天时燃料元件温度场的分布云图见图12(a). 图中所展示的平面为燃料板在厚度方向的对称面. 从 图12(a)可以看出, 燃料元件包壳部分的温度较低, 在

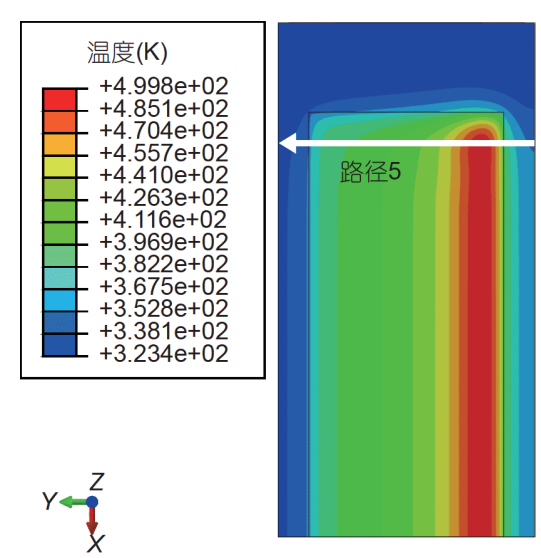

(a)

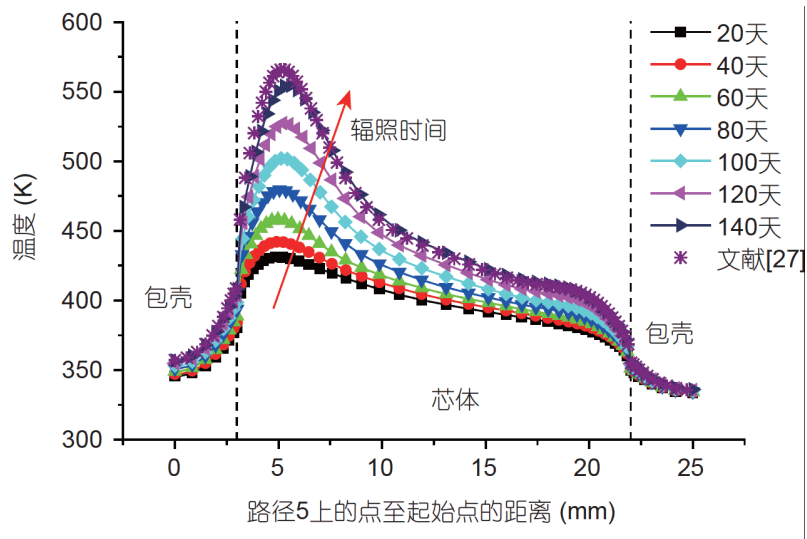

(b)

图 12 (网络版彩图)燃料元件温度场分布云图(a)和温度随辐照时间的演化结果(b)

Figure 12 (Color online) The contour plot of temperature (a) and the evolution results along Path 5 (b). 
323-400 K之间; 芯体部分的温度场沿宽度方向呈非均 匀分布, 最大值出现在靠近裂变率较大的一侧, 处于离 开芯体边界一段距离的位置; 芯体中心的温度为 $422 \mathrm{~K}$. 根据Kim等人 ${ }^{[8]}$ 的论文, 板L1P04A在辐照 98 天 时的中心区域温度为 $425 \mathrm{~K}$, 与本文计算结果非常相 近, 这也验证了本文建立的数值模拟方法的正确性.

选取过温度峰值、沿宽度方向的路径 5 , 输出不同 时刻的温度, 如图12(b)所示. 可以看出, 随着辐照时间 的增加, 路径起始点附近包壳部分的温度略有升高, 而 芯体部分的温度升高较为明显, 温度峰值的位置逐渐 向中心移动. 从 20-140天, 燃料元件温度场的最大值 由 $431 \mathrm{~K}$ 上升至 $554 \mathrm{~K}$, 增大了约 $28 \%$; 峰值出现的位置 沿路径 6 的方向移动了约 $0.5 \mathrm{~mm}$.

燃料元件温度场的分布规律受到芯体厚度增量、 产热率和材料热传导率的影响. 对于UMo合金芯体, 本文采用了Argonne实验室 ${ }^{[16]}$ 提出的热传导率模型, 该模型综合考虑了孔隙率和温度对芯体热传导率的影 响. 将芯体温度代入式(3)-(9), 并保持孔隙率Poro为 0 , 可以得到不考虑孔隙率情况下的芯体热传导率. 图13 展示了辐照 140 天时芯体热传导率沿路径 5 的分布情 况, 并对比了孔隙率对芯体热传导率的影响. 由于路径 5 两端为A16061包壳, 其热传导率在辐照过程中保持在 $180 \mathrm{~W} \mathrm{~m}^{-1} \mathrm{~K}^{-1}$ 左右, 远大于芯体热传导率, 故不在 图13中画出. 对比图13和12(b)可以看出, 在不考虑孔 隙率影响的情况下, 在温度最高的区域芯体热传导率 最高; 考虑孔隙率的影响后, 芯体温度最高的区域热 传导率反而较小. 结合图6(b)可以发现, 芯体温度最高 的区域具有较大的孔隙率, 降低了燃料芯体的热传导 率. 在不考虑孔隙率的情况下, 芯体热传导率最大值 为 $20.0 \mathrm{~W} \mathrm{~m}^{-1} \mathrm{~K}^{-1}$; 考虑率孔隙率后, 芯体热传导率最 大值为 $13.5 \mathrm{~W} \mathrm{~m}^{-1} \mathrm{~K}^{-1}$, 下降了 $32.5 \%$.

在本课题组早期的研究中 ${ }^{[27]}$, 裂变气体辐照肿胀 采用了 $\mathrm{Kim}$ 等人 ${ }^{[8]}$ 提出的经验公式, 而芯体热传导率 与孔隙率满足指数函数关系 ${ }^{[28]}$. 图 13 对比了辐照 140 天时两种热传导率模型的计算结果. 可以看出, 由 文献[27]模型得到的芯体热传导率略大于本文的计算 结果, 分布趋势也有所不同, 热传导率最大值出现在温 度最高的区域, 这跟所采用的裂变气体肿胀模型相关. 采用不同的裂变气体肿胀模型计算得到的芯体孔隙率 分布规律也不同. 如图6(a)所示, 本研究计算得到的芯 体孔隙率在离开芯体边界一段距离的位置达到较大

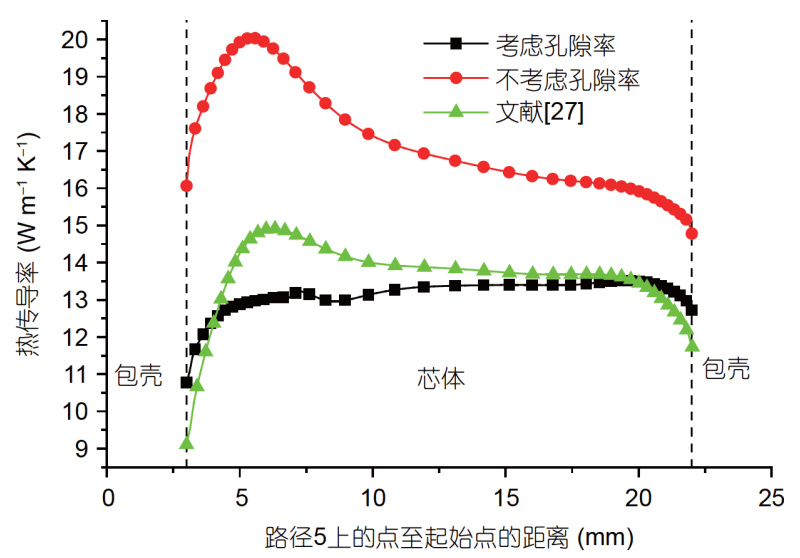

图 13 (网络版彩图)辐照 140 天时芯体热传导率沿路径 5 的 分布曲线

Figure 13 (Color online) The distribution curves of thermal conductivity along Path 5 on the 140th day.

值, 降低了这一区域的热传导率; 对比图9(a)和12(a)可 以看出, 采用裂变气体肿胀经验公式计算得到的芯体 孔隙率在燃料裂变率最大的一侧达到最大值, 故芯体 靠近界面的位置热传导率最小. 而在温度最高的区域, 孔隙率在 $19 \%$ 左右, 对热传导率的影响相对较小, 该区 域仍然具有较大的热传导率.

图12(b)对比了辐照140天时本研究和文献[27]模 型得到的燃料元件温度分布. 可以看出, 由文献[27]模 型计算得到的温度场峰值比本研究高约 $25.4 \mathrm{~K}$, 升高 了约 $5.0 \%$. 从图10(b)可以看出, 虽然对应的芯体热传 导率高于本文数值, 由于文献[27]模型计算得到的燃 料元件厚度增量大于本研究, 使燃料芯体在现时厚度 最大的局部区域产生了更多的热量, 不能快速地排出, 从而导致元件温度较高.

实际上, 由于包壳的热传导率远高于芯体的热传 导率, 主要由于芯体的厚度增大导致芯体的温度随着 辐照时间而增加.

\section{4 燃料元件的宏观界面正应力和界面微观正 应力}

图14(a)展示了燃料元件在辐照140天时的宏观界 面正应力云图, 图中展示的平面为芯体和包壳在厚度 方向上的界面. 可以看出, 界面拉应力主要出现在距 芯体边缘4-6 mm的区域, 其中界面拉应力的峰值出现 在芯体角落处. 选取包含峰值点、沿宽度方向的路径 6 , 输出不同时刻的宏观界面正应力. 从图14(b)可以看 


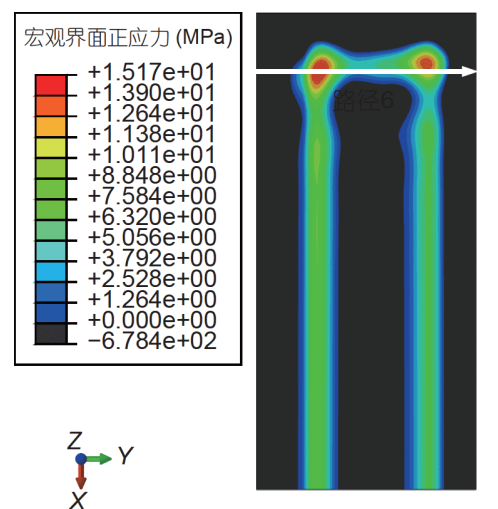

(a)

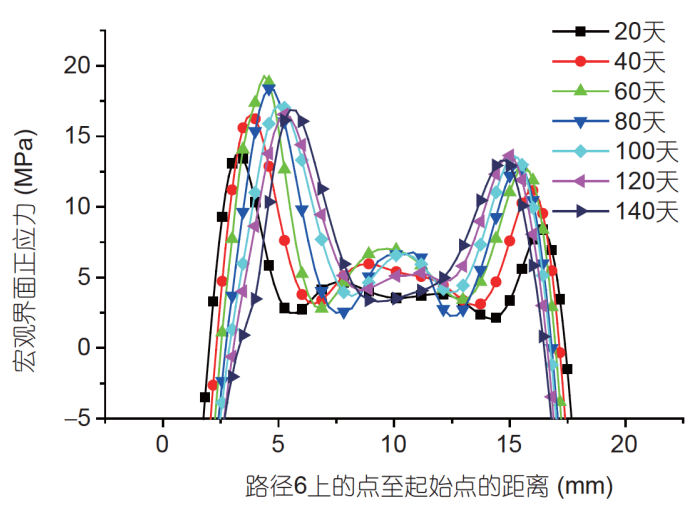

(b)

图 14 (网络版彩图)沿路径6宏观界面正应力分布云图(a)和宏观界面正应力随辐照时间的演化结果(b)

Figure 14 (Color online) The contour plot of interfacial normal stress (a) and its evolution results along Path 6 (b).

出, 随着辐照时间的增长, 宏观界面正应力的峰值先增 大后减小, 在第 60 天前后达到最大值, 约 $18.4 \mathrm{MPa}$; 峰 值出现的位置逐渐向芯体中心移动. 从20-140天, 宏观 界面正应力峰值向路径末端移动了约 $2.3 \mathrm{~mm}$.

由于在界面处产生了很多带孔压的孔洞，界面的 微观正应力不同于宏观界面正应力. 图15展示了界面 微观正应力随时间的演化规律, 其由式(24)计算得到. 可以看出, 随着辐照时间的增加, 界面微观正应力逐 渐增大, 峰值出现的位置逐渐向芯体中心移动. 辐照 140 天后，界面微观正应力的最大值可达到 $92 \mathrm{MPa}$ 左 右. 结合图6(b)可以发现, 由于燃料芯体的孔隙率随着 辐照时间逐渐增大，导致芯体与包壳的实际承载界面 的面积不断减小，所以，尽管宏观界面正应力呈现出

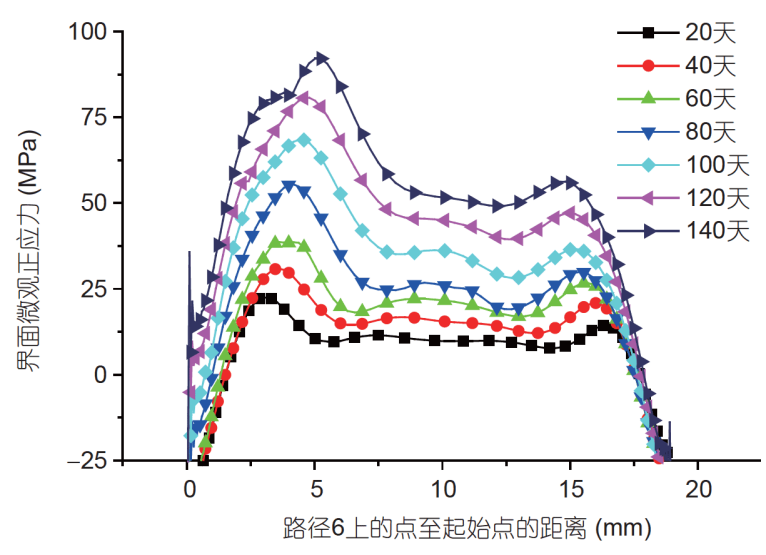

图 15 (网络版彩图)界面微观正应力随辐照时间沿路径6的 演化结果

Figure 15 (Color online) The evolution results of microscopic interfacial normal stress along Path 6.
先增大后减小的趋势，但界面微观拉应力随辐照时间 逐渐增大.

对比图14(b)和15可以看出, 相同时刻界面微观正 应力的峰值大于宏观界面正应力; 随着辐照时间的增 加, 两者差距逐渐增大, 辐照 140 天后, 界面微观正应 力的峰值比宏观界面正应力大 $77 \mathrm{MPa}$ 左右. 由此可见, 芯体与包壳的实际承载界面承受了较大的界面拉应 力, 可能会引起芯体与包壳界面的破坏. 而且, 还可以 发现, 路径起始点附近宏观正应力为负的点, 其界面微 观正应力也变为很大的拉应力. 如果在边缘处的界面 强度较弱, 则可能首先在靠近边缘处产生界面损伤及 破坏.

本文采用的孔压保持为 $100 \mathrm{MPa}$. 在燃料元件实 际服役过程中, 孔压会随着辐照时间和空间变化, 其 决定于孔隙率、释放到孔内的裂变气体原子数及温 度. 在燃料元件达到较高的燃耗后, 由于芯体内部出现 了较大的孔隙率和孔压, 界面微观应力可能达到很大 的数值, 并随着燃耗的发展进一步增长. 在较大的界 面微观应力的作用下, 芯体和包壳间可能会发生拉伸 破坏, 形成宏观裂纹, 如图1所示. 这一现象会威胁反 应堆的安全性, 影响燃料元件的使用寿命. 为了进一步 准确地计算界面微观正应力, 探究燃料元件界面破坏 机理, 需要进一步建立孔压随燃耗演化的理论模型, 深入分析界面微观应力的分布和演化规律. 通过前面 的分析可以看出, 实际的孔隙率决定于所使用的裂变 气体肿胀模型, 考虑温度及静水压力对裂变气体肿胀 的影响来对燃料元件界面破坏进行预测很关键. 


\section{5 热交换系数对燃料元件辐照热-力耦合行为的 影响}

在反应堆运行过程中, 由于冷却剂流速等参数的 影响, 燃料元件外表面的热交换系数会产生较大的变 化. 本节以 0.01 和 $0.035 \mathrm{~W} \mathrm{~mm}^{-2} \mathrm{~K}^{-1}$ 两种热交换系数 为例, 探究热交换系数对燃料元件辐照热-力耦合行为 的影响.

图16展示了不同热交换系数下路径 5 上各点在辐 照 140 天时的温度结果. 可以看出, 对于热交换系数较 小的燃料元件, 由于燃料板内积累的热量更多, 其温度 更高. 热交换系数 $0.01 \mathrm{~W} \mathrm{~mm} \mathrm{~m}^{-2} \mathrm{~K}^{-1}$ 下的芯体温度峰值 比 $0.035 \mathrm{~W} \mathrm{~mm}^{-2} \mathrm{~K}^{-1}$ 下的结果高约 $270 \mathrm{~K}$, 升高了 $50 \%$ 左右.

由于本文采用的裂变气体肿胀模型与温度相关, 温度场的变化会影响燃料芯体的裂变气体肿胀, 进而 影响芯体孔隙率. 图17展示了不同热交换系数下辐照 140 天时的芯体孔隙率. 可以看出, 不同热交换系数下 芯体孔隙率的最大值均出现在裂变率较大的一侧, 位 于离开路径起始点一段距离的位置, 但具体位置有所 不同. 热交换系数为 $0.01 \mathrm{~W} \mathrm{~mm}^{-2} \mathrm{~K}^{-1}$ 时, 其温度峰值 更靠近路径起始点, 且峰值比热交换系数为 $0.035 \mathrm{~W} \mathrm{~mm}^{-2} \mathrm{~K}^{-1}$ 的结果大 $3.7 \%$, 相对增长约 $15.4 \%$.

从式(24)可知, 燃料元件的界面微观正应力与芯 体孔隙率正相关. 图18展示了辐照140天时, 不同热交 换系数下路径 6 上各点的界面微观正应力. 可以看出, 在不同热交换系数下，界面微观正应力的最大值均出

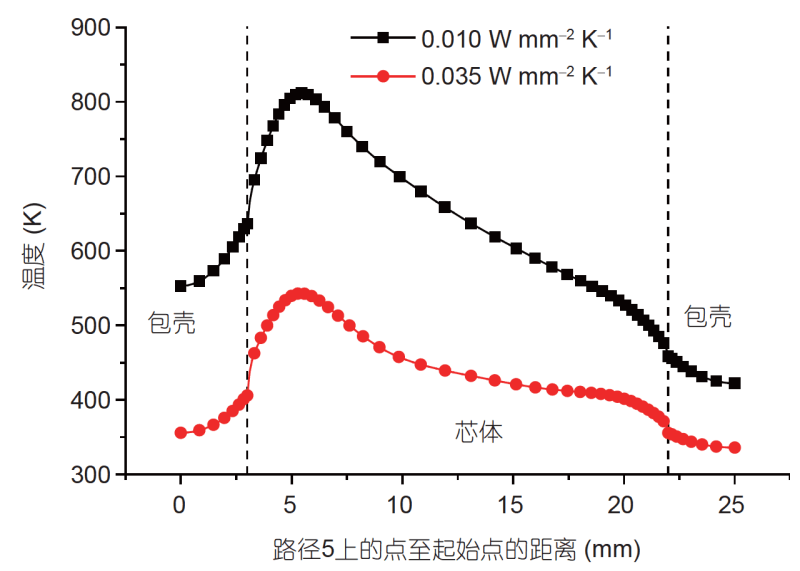

图 16 (网络版彩图)辐照140天时温度沿路径5的分布曲线 Figure 16 (Color online) The distribution curves of temperature along Path 5 on the 140th day.

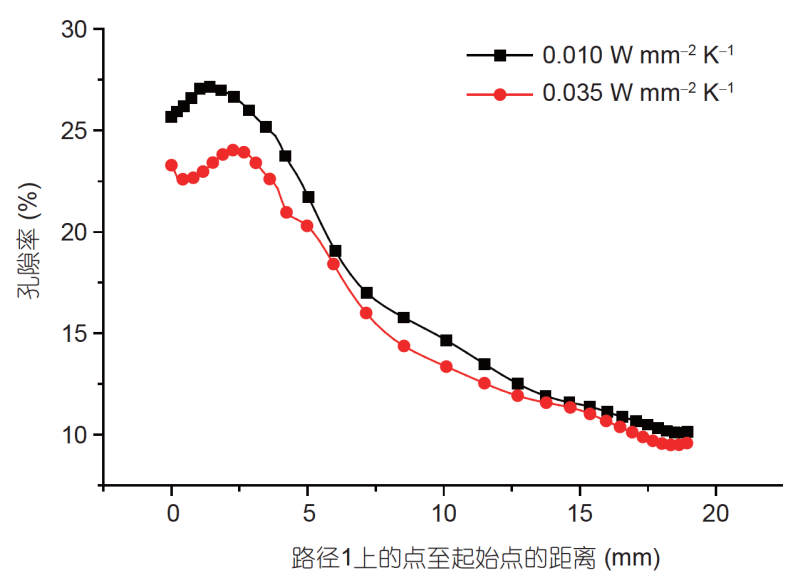

图 17 (网络版彩图)辐照 140 天时芯体孔隙率沿路径 1 的分 布曲线

Figure 17 (Color online) The distribution curves of fuel porosity along Path 1 on the 140th day.

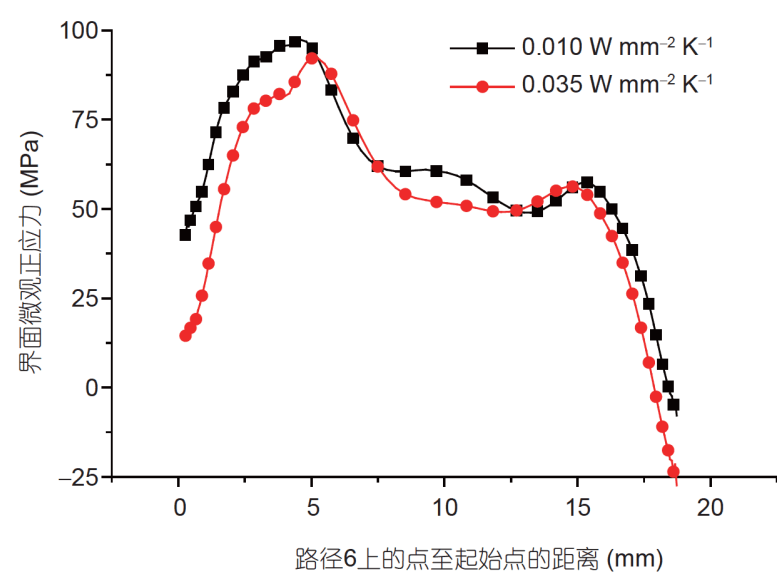

图 18 (网络版彩图)辐照140天时界面微观正应力沿路径6 的分布曲线

Figure 18 (Color online) The distribution curves of microscopic interfacial normal stress along Path 6 on the 140th day.

现在距路径 6 起始点 $5 \mathrm{~mm}$ 左右的位置; 热交换系数为 $0.01 \mathrm{~W} \mathrm{~mm}^{-2} \mathrm{~K}^{-1}$ 下, 具有更大的界面微观正应力, 其 峰值比热交换系数为 $0.035 \mathrm{~W} \mathrm{~mm}^{-2} \mathrm{~K}^{-1}$ 下的结果增大 约 $5 \%$.

本文在计算界面微观正应力时均采用 $100 \mathrm{MPa}$ 的 孔压. 实际上, 由于热交换系数为 $0.01 \mathrm{~W} \mathrm{~mm}^{-2} \mathrm{~K}^{-1}$ 下 的芯体温度更高, 其孔压应该大于热交换系数为 $0.035 \mathrm{~W} \mathrm{~mm} \mathrm{~K}^{-2}$ 下的数值. 为了更好地研究热交换 系数对燃料元件界面微观应力的影响, 需进一步建立 孔压随燃耗的演化模型，深入分析热交换系数对燃料 元件界面破坏行为的影响. 


\section{7 结论}

本文针对 $\mathrm{UMo} / \mathrm{Al}$ 单片式燃料元件，建立了对其辐 照热力耦合行为进行数值模拟的计算方法, 并建立了 孔隙率随燃耗演化的理论模型以及芯体/包壳界面微观 正应力的计算模型, 计算分析了不同热交换系数的燃料 元件在辐照过程中热力耦合行为, 探究了芯体孔隙率和 热交换系数的影响. 通过对燃料元件辐照过程中热-力 学场的分布及演化规律进行分析, 得到以下主要结论.

（1）随着燃耗的发展，UMo芯体的孔隙率逐渐增 大; 在低燃耗下，孔隙率峰值出现在芯体裂变率最大 的一侧，在高燃耗下，孔隙率的峰值出现在离开芯体 边界一段距离的位置. 在燃料板长度方向上的芯体/包 壳界面附近, 芯体孔隙率相对较低, 呈现出与其他区域 不同的分布规律，这主要由于裂变气体肿胀的静水压 力相关性导致.

(2) 随着燃耗的发展, 燃料元件厚度逐渐增大; 厚
度增量的峰值出现在靠近辐照强度最高的一侧、位于 芯体角落附近.

(3) 燃料元件的温度随着燃耗的发展逐渐升高; 温 度峰值出现在靠近裂变率最高一侧、离开芯体边界一 段距离的位置. 芯体最高温度随辐照时间逐渐增大, 主 要因为芯体的局部厚度逐渐增加.

（4）随着燃耗的发展，芯体/包壳界面宏观拉应力 峰值呈现出先增大后减小的变化趋势, 其峰值出现在 芯体角落附近, 数值不高. 在孔隙率和孔压的联合作 用下, 界面微观拉应力随着燃耗发展逐渐增大, 宏观 界面正应力为负的区域也会产生了较大的界面微观拉 应力, 芯体多孔结构及孔压是燃料元件界面破坏的主 要因素之一, 针对相关问题需要进行深入研究.

(5) 燃料元件表面热交换系数的降低会使芯体内 积累更多的热量，从而达到更高的温度，产生更大的 芯体孔隙率．在较大孔隙率的作用下，表面热交换系 数较小的燃料元件具有更大的界面微观正应力.

\section{参考文献}

1 Burkes D E, Casella A M, Huber T K. Modeling the influence of interaction layer formation on thermal conductivity of U-Mo dispersion fuel. J Alloys Compd, 2015, 618: 7-13

2 Burkes D E, Casella A M, Buck E C, et al. Development and validation of capabilities to measure thermal properties of layered monolithic U-Mo alloy plate-type fuel. Int J Thermophys, 2014, 35: 1476-1500

3 Burkes D E, Casella A J, Casella A M, et al. Measurement of fission gas release from irradiated U-Mo monolithic fuel samples. J Nucl Mater, 2015, 461: 61-71

4 Meyer M K, Wachs D M, Jue J F, et al. U.S. Progress in the development of very high density low enrichment research reactor fuels. In: European Reasarch Reactor Conference. Brussels, Belgium, 2012

$5 \mathrm{Hu} \mathrm{S}$, Burkes D E, Lavender C A, et al. Formation mechanism of gas bubble superlattice in UMo metal fuels: Phase-field modeling investigation. J Nucl Mater, 2016, 479: 202-215

$6 \mathrm{Hu} \mathrm{S}$, Burkes D, Lavender C A, et al. Effect of grain morphology on gas bubble swelling in UMo fuels - A 3D microstructure dependent booth model. J Nucl Mater, 2016, 480: 323-331

7 Sun R X, Xie H Y. Status and prospect of fuel development for research reactors (in Chinese). Atom Energ Sci Technol, 2011, 45: 847-851 [孙荣 先, 解怀英. 研究堆燃料的发展现状与前景. 原子能科学技术, 2011, 45: 847-851]

8 Kim Y S, Hofman G L. Fission product induced swelling of U-Mo alloy fuel. J Nucl Mater, 2011, 419: 291-301

9 Kim Y S, Hofman G L, Cheon J S, et al. Fission induced swelling and creep of U-Mo alloy fuel. J Nucl Mater, 2013, 437: 37-46

10 Cui Y, Huo Y, Ding S, et al. An analytical solution for simulation of the fission gas behaviors with time-dependent piece-wise boundary resolution. J Nucl Mater, 2012, 424: 109-115

11 Burkes D E, Casella A M, Casella A J, et al. Thermal properties of U-Mo alloys irradiated to moderate burnup and power. J Nucl Mater, 2015, 464: 331-341

12 Wachs D, Rice F, Glagalenko I. Blister Threshold Based Thermal Limits for the U-Mo Monolithic Fuel System. In: RERTR. Warsaw, Poland, 2012

13 Miller G K, Burkes D E, Wachs D M. Modeling thermal and stress behavior of the fuel-clad interface in monolithic fuel mini-plates. Mater Des, 
2010, 31: 3234-3243

14 Cui Y, Ding S, Chen Z, et al. Modifications and applications of the mechanistic gaseous swelling model for UMo fuel. J Nucl Mater, 2015, 457: 157-164

15 Zhao Y, Gong X, Ding S. Simulation of the irradiation-induced thermo-mechanical behaviors evolution in monolithic U-Mo/Zr fuel plates under a heterogeneous irradiation condition. Nucl Eng Des, 2015, 285: 84-97

16 Rest J, Kim Y S, Holmes G L. U-Mo Fuels Handbook Version 1.0. Technical Report. Argonne: Argonne National Lab. (ANL), 2009

17 Ozaltun H, Herman Shen M H, Medvedev P. Assessment of residual stresses on U10Mo alloy based monolithic mini-plates during hot isostatic pressing. J Nucl Mater, 2011, 419: 76-84

18 Dienst W. Irradiation induced creep of ceramic nuclear fuels. J Nucl Mater, 1977, 65: 1-8

19 Kim Y S, Jeong G Y, Park J M, et al. Fission induced swelling of U-Mo/Al dispersion fuel. J Nucl Mater, 2015, 465: 142-152

20 Zhao Y, Ding S, Huo Y, et al. Irradiation-induced thermomechanical behavior in ads composite fuel pellets: Mechanism and main influencing factors. J Thermal Stresses, 2016, 39: 630-657

21 Zhao Y, Ding S, Zhang X, et al. Effects of fuel particle size and fission-fragment-enhanced irradiation creep on the in-pile behavior in Cercer composite pellets. J Nucl Mater, 2016, 482: 278-293

22 Rest J, Hofman G L. An alternative explanation for evidence that xenon depletion, pore formation, and grain subdivision begin at different local burnups. J Nucl Mater, 2000, 277: 231-238

23 Spino J, Rest J, Goll W, et al. Matrix swelling rate and cavity volume balance of $\mathrm{UO}_{2}$ fuels at high burn-up. J Nucl Mater, 2005, 346: 131-144

24 Tian X, Kong X Z, Yan F, et al. Hydrostatic pressure effect of fission gas swelling in UMo/Al dispersion fuel plate (in Chinese). Atom Energ Sci Technol, 2017, 51: 2062-2068 [田旭, 孔祥吉, 严峰, 等. UMo/Al弥散燃料板内裂变气体肿胀的静压效应研究. 原子能科学技术, 2017, 51: 2062-2068]

25 Kong X, Tian X, Yan F, et al. Thermo-mechanical behavior simulation coupled with the hydrostatic-pressure-dependent grain-scale fission gas swelling calculation for a monolithic UMo fuel plate under heterogeneous neutron irradiation. Open Eng, 2018, 8: 243-260

26 Gao L J, Chen B D, Jiang S Y, et al. Analysis of blistering mechanism for dispersion-type fuel plates during irradiation (in Chinese). Atom Energ Sci Technol, 2012, 46(Suppl): 819-825 [高利军, 陈炳德, 姜胜耀, 等. 弥散型燃料板的辐照起泡机理分析. 原子能科学技术, 2012, 46(增刊): 819-825]

27 Yan F, Zhao Y, Ding S. Effect of fuel meat thickness on the non-uniform irradiation-induced thermo-mechanical behavior in monolithic UMo/Al fuel plates. In: ICONE. Shanghai, 2017

28 Ryu H J, Kim Y S, Park J M, et al. Performance evaluation of U-Mo/Al dispersion fuel by considering a fuel-matrix interaction. Nucl Eng Tech, 2009, 40: 409-418 


\title{
Effects of porous fuel structure on the irradiation-induced thermo-mechanical coupling behavior in monolithic fuel plates
}

\author{
YAN Feng ${ }^{1}$, KONG XiangZhe ${ }^{1}$, DING ShuRong ${ }^{1 *}$, HE DaMing ${ }^{2}$, LI YuanMing ${ }^{2}$, \\ CHEN Ping ${ }^{2} \&$ ZHOU Yi ${ }^{2}$ \\ ${ }^{1}$ Institute of Mechanics and Computational Engineering, Department of Aeronautics and Astronautics, Fudan University, \\ Shanghai 200433, China; \\ ${ }^{2}$ Science and Technology on Reactor System Design Technology Laboratory, Nuclear Power Institute of China, Chengdu 610213, China
}

A UMo/Al monolithic fuel plate is composed of UMo fuel meat and Al cladding. These fuel plates with a high uranium density have a promising prospect to be used in advanced research and test reactors. Under the neutron irradiation environments, nuclear fissions occur to result in heat generation in the fuel meat; accumulation of fission-induced solid and gaseous products will lead to irradiation swelling; irradiation creep of UMo will affect the mechanical interactions between the fuel meat and the cladding. Especially, intragranular and intergranular gas bubbles will make the fuel meat evolve into a porous structure, and the fuel porosity and pore pressure will change continuously with the irradiation time. As a result, a complex irradiation-induced multi-scale thermo-mechanical coupling behavior needs to be simulated for optimal design and safety evaluation of UMo/Al monolithic fuel plates. In this study, a theoretical model for fuel porosity evolution is developed, based on a fission gas swelling model with the grain recrystallization, the resolution of intergranular gas atoms and the dependence of hydrostatic pressure involved. Moreover, the computation method of microscopic interfacial normal stress is established, with consideration of fuel porosity and pore pressure. The evolution model of fuel porosity is introduced into three-dimensional finite element simulation of the irradiation-induced multiscale thermo-mechanical coupling behavior, which correlates the current thermal conductivity of UMo fuel with the fuel temperature and porosity. The distribution and evolution disciplines of thermo-mechanical variables in the fuel plate are obtained at different irradiation time. In addition, the effects of fuel porosity on the temperature, the main deformation and microscopic interfacial normal stresses are investigated. Simultaneously, the effects of surface heat transfer coefficient on the thermo-mechanical behavior are analyzed. The research results indicate that the porous fuel structure and fission-gas induced pore pressure become the main fracture mechanisms of monolithic fuel plates.

monolithic fuel plate, neutron irradiation, fission gas swelling, irradiation-induced creep, porous structure, interfacial fracture mechanism

PACS: $24.75 .+\mathrm{i}, 44.10 .+\mathrm{i}, 46.15 .-\mathrm{x}$

doi: 10.1360/SSPMA2018-00297 